\title{
Vécu et perception de la frontière entre la RDA et la RFA
}

Experience and feeling of theformer German-German border

Erlebnis und Wahrnehmung der ehemaligen innerdeutschen Grenze

Heidi Armbruster et Ulrike H. Meinhof

Traducteur : Bertrand Abadie

\section{(2) OpenEdition}

Journals

Édition électronique

URL : http://journals.openedition.org/rge/2213

DOI : $10.4000 /$ rge.2213

ISSN : 2108-6478

Éditeur

Association des géographes de l'Est

Édition imprimée

Date de publication : 1 septembre 2003

ISSN : 0035-3213

Référence électronique

Heidi Armbruster et Ulrike H. Meinhof, « Vécu et perception de la frontière entre la RDA et la RFA », Revue Géographique de l'Est [En ligne], vol. 43 / 4 | 2003, mis en ligne le 19 novembre 2010, consulté le 08 septembre 2020. URL : http://journals.openedition.org/rge/2213 ; DOI : https://doi.org/10.4000/ rge. 2213

Ce document a été généré automatiquement le 8 septembre 2020

Tous droits réservés 


\title{
Vécu et perception de la frontière entre la RDA et la RFA
}

\author{
Experience and feeling of theformer German-German border \\ Erlebnis und Wahrnehmung der ehemaligen innerdeutschen Grenze
}

Heidi Armbruster et Ulrike H. Meinhof

Traduction : Bertrand Abadie

\section{Introduction}

Le film de Sinan Cetin, "Propaganda ", est sorti en 1999. Bien que mis en scène dans l'est de la Turquie en 1948, sa représentation ironique de ce qui peut arriver aux individus d'une communauté divisée par une frontière imposée par l'extérieur servira de point de départ à notre réflexion sur une région frontalière très différente de l'Allemagne de l'après-guerre. Dans la séquence d'ouverture de la fiction de Cetin, des villageois en habits de fête attendent à côté d'une gare ferroviaire en plein milieu d'une grande plaine aride et jaunie par la chaleur sèche de l'été. Tout le monde attend avec enthousiasme l'arrivée de Mehdi, un bon père de famille du village de retour après une longue absence à la lointaine capitale, Ankara. Mehdi, vêtu d'un uniforme de chef de douane et accompagné de soldats et d'hommes en costume, reçoit un accueil chaleureux. Sous le regard perplexe de tous, les soldats déchargent du train de grands rouleaux de fil barbelé. En temps utile, ils construisent une clôture qui sépare le village en deux, divisant alors un espace qui, auparavant, ne faisait qu'un.Dès lors, ceci représente une réelle «frontière » dotée d'un bureau de chef de douane et d'un portail frontalier. Comme Mehdi ne se lasse jamais de le souligner, cette "frontière", scrupuleusement surveillée jour et nuit, incarne « l'Etat». Les sceptiques sont informés que les «mesures viennent d'Ankara.» Bien que Mehdi lui-même ait du mal à accepter ce décret venant de la « grande capitale » - après tout, son meilleur ami, le docteur du village, vit désormais en dehors "de l'Etat" - la frontière crée bientôt sa propre dynamique, divisant progressivement les individus en différents groupes se considérant les uns les autres avec une suspicion grandissante. Alors que les villageois 
sont dépourvus de passeports pour traverser la frontière dans un sens ou dans l'autre, une série d'étranges évènements survient: l'instituteur du village ne peut pas accéder à l'école, la prostituée locale est séparée de ses clients, et le berger ne peut plus conduire son troupeau de l'autre côté de la frontière. Le drame prend plus d'ampleur alors que l'animosité et la violence entre les deux côtés de la frontière s'intensifient. On en vient même à tirer sur quelqu'un.

2 Le film, drôle et ironique, aborde quelques vérités élémentaires concernant les frontières d'Etats : elles sont des « lieux et symboles de pouvoir » (Donnan \& Wilson 1999), elles peuvent apparaitre dans la nuit, et peuvent empiéter de manière profonde et extrêmement perturbatrice sur la vie des individus.

3 Dans le film de Cetin, c'est l'histoire de deux amoureux séparés qui rend visible l'impact de la frontière sur la société, et qui plus est produit la force suffisante pour la détruire. Le film se termine alors sur la vision utopique du monde du privé - l'amour - venant à bout du pouvoir public de l'Etat distant et abstrait.

4 Parmi les témoignages sur la frontière que nous présentons dans ce chapitre, peu font référence à des histoires d'amour et les fins sont relativement heureuses. De plus, nos propres témoignages montreront, tout comme dans le film, que la création artificielle d'une frontière et par là, d'une division forcée qui sera lentement acceptée, obligera les individus à réadapter et remodeler la carte cognitive de leur espace de vie et de leur rapport aux autres. Tant et si bien que même une décennie après la réunification, la séparation ayant existé pendant 40 ans demeure un point de référence non seulement pour les récits du passé mais également pour les récits de la vie actuelle. En nous appuyant sur des interviews menées en 2000 auprès de trois générations d'individus nés des deux côtés de la frontière séparant les Allemagnes, nous retracerons la conceptualisation de la frontière à travers le récit du déroulement chronologique d'évènements survenus dans la vie d'individus et de leur famille.

\section{Sur la plage claire de la Saale : description géopolitique d'une ancienne frontière allemande}

Ce projet traite des identités frontalières de l'ancienne séparation des deux Allemagnes. Notre étude a visé la région limitrophe des États fédéraux de la Thuringe, la Saxe et la Bavière. Lorsqu'en 1945 les Alliés dessinèrent la frontière qui divisait le pays, ils le firent sur la base de régions allemandes historiquement distinctes mais néanmoins liées. De fait, la Bavière fut occupée par l'armée américaine alors que la Thuringe le fut par l'armée soviétique. La limite les séparant devint rapidement une frontière de plus en plus strictement gardée entre deux Etats distincts, tant au niveau du modèle idéologique et économique, que de celui du niveau de vie. Lors de la réunification en octobre 1990, la RDA fut dissoute, la Thuringe et la Saxe furent refondées en unités fédérales, et l'ancien système administratif fut totalement remanié sur le modèle de la RFA.

6 Entre 1945 et 1989/90, la région nous intéressant pour cette étude faisait partie d'un axe de conflit idéologique entre l'«Est » et l'«Ouest» devenu si obstiné que, depuis cette date, les populations locales se considèrent comme étant soit « de l'Est », soit « de l'Ouest ». Cependant, il est intéressant de préciser qu'avant 1945, cette région était économiquement, socialement et culturellement intégrée, avec des flux économiques et 
culturels circulant suivant un axe Nord-Sud. Nuremberg en Moyenne-Franconie, Hof en Haute-Franconie, Dresde en Saxe et Wroclaw, désormais polonaise mais anciennement appelée Breslau et située dans la province allemande de Silésie, formaient un axe historique d'échanges commerciaux. La plupart des matières premières utilisées dans les industries franconiennes de la porcelaine, du verre, du cuir ou du papier venaient de Saxe, de Bohême, de Thuringe ou de provinces anciennement est-allemandes. De la même façon, beaucoup de biens produits en Bavière étaient acheminés vers le Nord plutôt que vers le Sud (Maier, 1990). Ce fait fut clairement confirmé par nos informateurs bavarois les plus âgés qui se rappellent tous avoir eu une orientation économique, culturelle et sociale vers la Thuringe et la Saxe avant 1945 : les gens y travaillaient souvent, y faisaient leurs courses, et y entretenaient de proches liens sociaux. L'érection et la militarisation croissante de la frontière furent alors les raisons qui mirent un terme à ces pratiques. Dès lors, les deux côtés furent incorporés dans deux systèmes différents, voire opposés: à l'Ouest, les principes de démocratie parlementaire, de fédéralisme et d'économie de marché capitaliste, et à l'Est, le Centralisme Démocratique, le marxisme-léninisme et l'économie à planification centralisée.

7 La Thuringe et la Bavière (Haute-Franconie) dans lesquelles nous avons mené nos interviews, forment une région dotée de villages et de petites villes ne comptant pas plus de 2000 habitants. Cette façon d'occuper le territoire est de plus restée la même durant les années de la division de l'Allemagne, ce qui ne fut pas le cas de l'agriculture de cette région qui subit, quant à elle, un certain nombre de changements. En Thuringe, les fermes devinrent collectives et une grande coopérative agricole (la LPG) fut créée. De plus, une usine de cuir située sur les bords de la rivière Saale fournit du travail à plusieurs centaines de personnes durant les années d'existence de la RDA, et (tous) les individus d'âge moyen ou plus âgés interviewés avaient tous travaillé à l'usine ou à la LPG. Après 1990, la LPG fut transformée en entreprise commerciale et l'usine de cuir fut fermée et, finalement, démontée.

8 Du côté de la Haute-Franconie, les fermes de tailles petites et moyennes restèrent intactes même si, comme toute communauté agricole de l'Union européenne, elles faisaient face à de plus en plus de problèmes financiers. Au moment de notre étude en 2000 , seules deux ou trois grandes fermes s'étendant sur plusieurs villages demeuraient en pleine activité et en plein processus de spécialisation et de rationalisation de leur production. Durant les années 60 et 70 , la région des deux côtés de la frontière subit une baisse de population, alors que de nombreux jeunes partaient en quête de meilleures opportunités professionnelles dans de plus grandes villes (Weber, 1990). De plus, une partie de la population le long de la frontière en Thuringe fut expulsée ou évacuée par le régime de la RDA de cette zone d'intenses contrôles et de restrictions. En Haute-Franconie (Allemagne de l'Ouest), ce déclin de la population continua jusque vers la fin des années 80 et ne fut stoppé que lorsqu'un nombre conséquent d'Allemands de l'Est vinrent s'installer dans la région. Depuis 1993, nombreux sont ceux qui traversent la frontière dans les deux sens pour travailler ou profiter des infrastructures commerciales de plus grandes villes. Bien que cette région ne soit plus géographiquement marginale, l'ensemble de la population locale connait des difficultés économiques et le processus de «rapprochement » mental et culturel est toujours en cours. 
D'un point de vue physique, la région a toujours semblé être un espace unifié. L'impression que le spectateur s'en fait est influencée par sa mosaïque de plateaux, de vallées fluviales et plus particulièrement celle de la rivière Saale, de collines arrondies et souvent très boisées, de grands prés et de champs, de routes sinueuses reliant un grand nombre de petits villages entre eux. La région est attrayante - la beauté de la rivière Saale est par exemple le thème de plusieurs chansons folkloriques célèbres - et depuis la réunification, les communautés locales essayent à nouveau de la promouvoir pour le tourisme. Les personnes interrogées vivent dans cinq communautés villageoises du côté de la Thuringe, et dans quatre communautés de la Haute-Franconie. De plus, exception faite de Hirschberg en Thuringe qui compte environ 2000 habitants, toutes les autres communes étudiées n'en comptent qu'entre 40 et 250. Ces villages enjambent la Saale, d'autres sont situés au cœur même de la vallée, et d'autres perchés sur les hauts plateaux de part et d'autre. La Saale prend sa source dans le Fichtelgebirge bavarois, puis se dirige vers le nord, à travers la Thuringe, pour se jeter dans l'Elbe après un parcours de $426 \mathrm{~km}$. Une partie de la frontière entre la Thuringe et la Bavière suit le cours de la rivière, formant de la sorte une limite «naturelle» entre les deux Etats allemands. Même si la frontière ne suit la rivière que sur une courte partie de son cours, les exemples étudiés montrent à quel point elle a été importante dans la carte cognitive de la vie frontalière des individus.

Figure 1 : Entre Bavière et Thuringe, la région de l'ancien rideau de fer entre RFA et RDA.

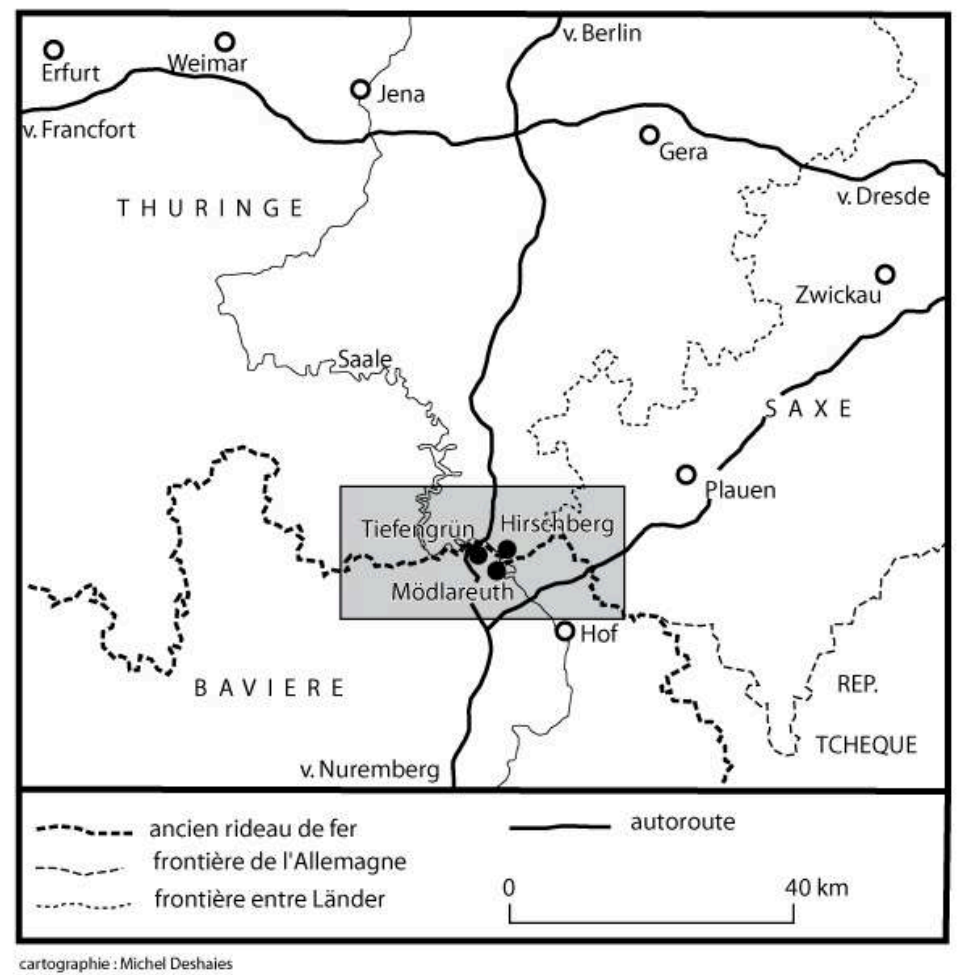

\section{La frontière comme carte cognitive}

10 Tout comme dans la représentation faite de la frontière fictive et de l'évolution progressive des perceptions transfrontalières des individus dans le film de Cetin, nous 
avons relevé une gamme de perceptions elles-mêmes changeantes au travers des témoignages des divers interlocuteurs. Ces dernières correspondaient à différentes phases chronologiques dont nous avions en partie fait resurgir le souvenir en présentant des clichés illustrant différentes périodes de manière évidente (Meinhof \& Galasinski 2000). Même si ces témoignages nous ont été apportés en 2000, il n'en demeure pas moins évident que leur contenu narratif établit un lien entre les expériences de la vie quotidienne et les politiques publiques, la propagande au sein même du pays, un régime frontalier s'affermissant, et des changements économiques et culturels tout au long de la vie de la population à ce moment-là. La présentation de nos données dans un cadre chronologique qui suit plus ou moins les 5 étapes de la démarcation (depuis les années faisant immédiatement suite à la guerre, jusqu'à la fin $\mathrm{du}$ siècle) permettra de nous concentrer sur quelques uns des principaux points de repère conceptuels dans les témoignages sur la démarcation.

11 De manière évidente et naturelle, ces récits sont ceux de la mémoire, sélectionnée et construite à travers le langage de chacun des témoins. Pour autant, ce ne sont pas uniquement des anecdotes appartenant à des individus particuliers, mais plutôt la preuve d'un répertoire culturel commun à tous ceux ayant vécu le long de la démarcation. Nous avons déjà abordé dans d'autres travaux un certain nombre de données ici présentes (Meinhof \& Galasinski 2000 ; 2002 ; Galasinski \& Meinhof 2002 ; Armbruster \& Meinhof 2002; Armbruster \& Meinhof in press ; Armbruster \& Meinhof forthcoming). Dans ce chapitre, nous nous intéressons en particulier aux récits percevant la frontière comme un monument, une composante géographique devenue élément quotidien et symbolique du paysage des individus. L'important ici est de remarquer les étapes suivant lesquelles un élément étrange et difficilement gérable au départ, devient progressivement ordinaire et "naturel » jusqu'au plus loin dans la construction de la mémoire des gens. L'adaptation et la naturalisation sont des procédés humains banals et omniprésents qui n'auraient rien de remarquable s'ils n'intervenaient pas dans un si grand contexte géopolitique. Le dessin de la frontière entre les deux Allemagnes trouve ses racines dans un contexte géographique plus large défini par des guerres et des conflits ayant bouleversé les lois du droit et de l'appartenance, bouleversement qui semble avoir fortement marqué les frontaliers. De fait, comprendre comment leurs récits reflètent et construisent ces modifications perturbatrices nous donne une meilleure connaissance de ce qu'étaient les interactions quotidiennes entre les individus et de ce qui leur était imposé par l'État. En d'autres termes, nous espérons faire la lumière sur une relation entre une géographie tangible d'appartenance imposée et/ou niée par des États, et une géographie subjective du lieu et d'appartenance vécue par nos interlocuteurs. Notre compréhension de la géographie dans ce contexte identifie une relation entre les individus et les lieux de deux types différents, d'un côté les pratiques sociales et les expériences du lieu, et de l'autre, les conditions physiques et matérielles qui déterminent la vie de chacun (Rogoff 2000).

12 L'apparition de la frontière, ses quarante ans de présence, et sa disparition sont un témoignage du caractère social et transformateur inhérent à la géographie et au « lieu ». Un grand nombre de géographes et de chercheurs en théories culturelles ont d'ailleurs redéfini la notion de lieu. De fait, plutôt que de concevoir les lieux comme des unités territoriales individuellement définies en des termes historiques, culturels et physiques, ils les considèrent en terme d' "activité »; un endroit serait donc marqué par des entrecroisements d'activités bien particulières, « de connections et relations, 
(...) d'influences et de mouvements" (Massey, 1995). Les endroits sont donc " construits» par des groupes de relations sociales leur attribuant leur individualité. Cette vision est de plus très évidente dans les récits de nos interlocuteurs lorsque ceuxci décrivent l'importation de la frontière dans leur espace de vie local et sa présence parmi eux en des termes purement sociaux. Ce qui fit de la frontière une réalité pour eux fut la transformation des relations sociales, l'absence de certains produits, l'afflux militaire, la fuite de certains locaux, l'échange de salutations en silence, la perte du lieu de travail de l'autre côté de la rivière, ou encore les visites familiales dominicales rendues impossibles. Ces activités et expériences affectèrent leur manière d'appréhender leur espace et de s'y déplacer, mais aussi l'approche de leur propre identité. L'érection de la frontière, en soi un acte social, se résumait à tenter de redéfinir et de «refaire » le lieu, et établissait un lien tout à fait tangible entre les individus locaux et les pouvoirs politiques des localités éloignées. Nos exemples mettront ici en relief certains des efforts mis en œuvre pour imposer et gérer les nouveaux sens des lieux comme deux sphères nationales et idéologiques antagonistes. Ils feront aussi référence aux problèmes en essayant d'annuler l'antagonisme de ces identités spatiales, bien vivaces quarante ans plus tard.

\section{Les années consécutives à la guerre : « nous avions alors encore une frontière agréable »}

13 La ligne de démarcation entre la Thuringe et la Bavière fut au départ gardée par les forces d'occupation américaines et russes. La plupart des ponts sur la rivière de la Saale avaient alors été détruits dans un dernier effort allemand visant à freiner la progression des armées étrangères.

Les récits traitant de cette période parlent de maintenir le contact avec l'autre côté, et décrivent souvent des traversées de la rivière, d'un champ ou d'une forêt, et par là, la défiance des gardes américains et russes. Anna Weiss est une Bavaroise de 70 ans aujourd'hui qui a travaillé en Thuringe jusqu'en 1948. Tout en nous racontant l'histoire dramatique de son trajet à pied, long de quelques kilomètres pour se rendre au travail, elle exprime sa propre compréhension de cette période déroutante et angoissante :

« Nous n'avions alors pas de bicyclettes. Il nous fallait marcher durant tout le trajet. Parfois, nous prenions le chemin de la forêt car il y avait un raccourci, au moins pendant l'été, car l'hiver c'était impossible. Combien de fois ai-je marché de D. à M. à travers la forêt, sans rien y voir, absolument rien, très lentement, et tout d'un coup, à côté de vous, un cri «stoj»! Puis il fallait marcher avec eux et leurs mitrailleuses. Mais personne ne m'a jamais fait de mal. Je disais toujours «moi ROBOTA, au travail, et heu, "voici des papiers russes". Ils prenaient parfois les cartes d'identité qu'ils regardaient, souvent dans le mauvais sens, à la lumière d'un briquet ou d'une allumette. Mais "dawaj dawaj !" vite, vite, je devais me dépêcher pour rentrer à la maison, disaient-ils. Oui, et dans le village aussi il y avait des contrôles et tout le reste. Vous aviez peur et étiez heureux une fois que vous les aviez passés. "

Eva Tischler, qui est du même âge qu'Anna, raconte des expériences similaires d'une perspective thuringienne. Elle se souvient passer furtivement à côté des contrôles russes pour aller acheter des produits en Bavière ou aller à des bals de l'autre côté et, une fois là-bas, de duper les contrôles américains pour pouvoir retrouver des amis dans une auberge. Des récits identiques décrivent le partage de la même école, des échanges 
de marchandises, des rendez-vous de couples d'amoureux, et même des visites régulières ou la contrebande à travers la rivière Saale ; les gens se souviennent des pieds mouillés, des informations criées et des choses lancées de l'autre côté. Plus que toute autre chose, ils se souviennent des noms et des maisons (certaines démolies depuis bien longtemps) de ceux de l'autre côté. Ces histoires contiennent humour, bravade, nostalgie et un sentiment clair d'appartenir à une même entreprise collective. Tous ont en commun cette même expérience de difficulté et pénurie économique d'après-guerre et de collaboration pour défier la ligne de démarcation.

\section{Le début des années 50 : « et tout à coup, tout fut terminé »}

16 Les deux Etats allemands furent fondés en 1949. Entre 1950 et 1951, 360000 personnes s'échappèrent à l'Ouest, et en 1952, la RDA décida de renforcer la sécurité à la frontière et $d$ 'y établir des zones d'accès restreint (BMIB, 1989). Ces zones étaient des bandes de $5 \mathrm{~km}$ de large, militairement protégées, et interdites aux non-résidents. Les résidents quant à eux avaient besoin d'autorisations spéciales devant être présentées aux gardes frontaliers à chaque allée et venue en dehors de leur zone. C'est en 1952 qu'eut lieu la première vague de déménagements forcés en dehors de la zone, au cours de laquelle des citoyens soi-disant " politiquement déloyaux» durent partir durant la nuit pour s'installer dans l'arrière-pays. Toutes les communautés de la Thuringe que nous avons étudiées perdirent des citoyens de cette façon-là. Les gens allant encore vers la RDA pour des raisons professionnelles durent y mettre un terme en 1952 (BMIB, 1989).

Les récits relatant cette période indiquent un sentiment de choc et de danger.

Gunter Bauer, né en 1937, vécut son enfance du côté bavarois de la Saale et déménagea à l'âge de 12 ans chez des parents habitant de l'autre côté en Thuringe. À la fermeture de la frontière, il lui fut ensuite impossible de rendre visite à sa mère. Dans son récit décrivant cette période, Gunter se visualise lui-même d'un côté de la frontière en train d'essayer de communiquer avec des membres de sa famille.

«Mon oncle n'ayant pas d'enfants, mon grand-père décida que la maison devait me revenir, donc à l'âge de 12 ans, je suis venu ici. Je suis allé à l'école ici pendant deux ans, puis je me suis marié et ai eu deux enfants. Et oui, en effet, il y avait le problème de ma mère se tenant là-bas, on n'était pas supposé crier, mais on le faisait quand même. Bon, et plus tard dans la soirée, les services de sécurité de l'Etat venaient chez vous... on vous informait toujours, vous savez, hé bien, on ne pouvait pas parler doucement, il fallait [crier de l'autre côté de la rivière]. Et le village tout entier pouvait entendre, c'était ces temps-là, c'était bizarre. Bon, c'est fini maintenant $»$.

Gunter était visiblement ému en disant cela mais il eut du mal à trouver les mots justes pour décrire son expérience, devint mal à l'aise et s'interrompit. Son sentiment d'appartenance avait été douloureusement perturbé, et contrastant avec le récit cidessus, l'Etat apparaît clairement contrôleur et menaçant. Gunter mentionne le fait d'avoir été dénoncé par des villageois, une claire référence à l'esprit de communauté brisé dans ce village de Thuringe.

Beaucoup de Bavarois interrogés se rappelèrent cet état de choc et l'éveil forcé sur la finalité de la frontière alors qu'ils racontaient la fuite in extremis d'une famille dont la maison venait juste d'être transformée en forteresse pour les besoins de la frontière. 
Même ceux qui n'avaient pas été témoins de la scène en parlaient avec agitation, n'omettant pas de mentionner l'image des gardes frontaliers armés de la Thuringe sur le point de faire une descente dans la maison alors que ses occupants étaient en train de sauter par la fenêtre du côté qui était désormais l'Ouest. Armés de la même façon, les gardes bavarois reçurent les réfugiés. Dans les deux cas, des histoires visuellement fortes suscitèrent des sentiments de tristesse, de douleur, et de peur qui commencèrent alors à donner son sens à la démarcation.

\section{Fin des années 50 - début des années 60 : « ce que nous avons vécu était incroyable »}

En 1957 la RDA publia un décret selon lequel il était désormais illégal de quitter le pays et augmenta de plus en plus le nombre des restrictions pour se rendre en RFA. La sécurité fut alors accrue, et en 1961 la frontière était devenue un monument armé de tours de gardes, de bunkers, de rangées de clôtures, de projecteurs et de chiens de garde. De plus, beaucoup des maisons proches de la clôture de démarcation furent démolies.

Les récits de cette période traitent de plus en plus du régime frontalier de la RDA et les Thuringiens y décrivent comment la démarcation, en tant que barrière physique, infiltra leurs vies quotidiennes et comment ils furent en quelque sorte forcés de revoir leur approche de l'espace environnant et du déplacement en son sein. De plus, ces récits et anecdotes qualifient le régime frontalier d'absurde et dans tous les cas, celui-ci aura eu de graves conséquences sur les relations inter-frontalières. La population de part et d'autre se connaissant toujours est de plus en plus contrainte de perdre le contact.

Jospeh Seiler est né en 1931 et vécut toute sa vie dans un village de fermiers de Thuringe. Lorsque les fermes furent collectivisées, il commença par travailler dans les étables de la coopérative, et l'une de ses tâches était de conduire les troupeaux pour aller paître le matin et de les ramener le soir. Les pâturages se situaient le long de la frontière et donnaient sur le côté bavarois.

«La frontière était alors une installation des plus simples, et non déjà faite de clôtures de fils de fer barbelés, mais nous n'étions malgré tout pas autorisés à la traverser. Je travaillais dans le champ qui se trouvait exactement le long de la démarcation. C'était un dimanche, une très belle journée où il faisait très chaud. Deux jeunes gars arrivèrent à moto en provenance du côté bavarois et s'arrêtèrent à une vingtaine de mètres de moi. Ils me dirent bonjour. "Comment allez-vous ?" Je leur répondis : “Ça va bien. Je m'en sors" et ainsi de suite. "Belle journée aujourd'hui, n'est ce pas ?" et je leur répondis : “Oui, c'est agréable mais je commence à avoir soif maintenant". "Nous pouvons t'aider à ce niveau-là" Je ne pouvais pas savoir qu'ils avaient de la bière dans leurs sacs. Ils me lancèrent une bouteille. "Et on dirait que tu n'as rien à fumer non plus ?" "Je ne fume pas." mais ils me jetèrent des cigarettes aussi. Or, des gardes positionnés près de la forêt virent tout. Une fois que les deux gars sur la moto furent partis, ils s'avancèrent vers moi. "Que s'est-il passé ici ?" "Hé bien je suis sûr que vous le savez bien. Ils m'ont lancé deux bouteilles de bières et deux paquets de cigarettes." "Tenez" je leur dis "Les voilà", "Et vous avez ouvert une des bouteilles? Mais vous n'êtes pas autorisé à faire ça." Alors je leur ai dit "J'avais soif, voilà pourquoi." "Vous n'aviez pas le droit de faire ça, nous devons donc faire un rapport" ». 
Joseph poursuit son récit en décrivant comment il fut escorté à travers le champ par les deux officiers et leurs mitrailleuses jusqu'au commandant au village.

«Durant 45 minutes il ne fit que me lire le règlement frontalier.» Ce que Joseph considérait être alors un comportement social ordinaire avait été interprété par les officiels comme une atteinte à la sûreté de l'Etat. Il est fort probable que ceci resta la dernière communication verbale avec l'autre côté de la démarcation.

Eva vivait dans un village de la vallée de la Saale. De la même façon, elle décrit comment les contacts avec l'autre côté devinrent de plus en plus impossibles.

« Nous avions pour habitude d'aller nous asseoir dans le jardin derrière la maison tous les dimanches, mais aussi là et là-bas où ceux de l'Ouest étaient. Tout le monde se connaissait car bien sûr il y avait là deux villages l'un à côté de l'autre et beaucoup avaient fui [à l'Ouest]. Le dimanche nous nous faisions tous des signes de loin. C'était alors le bon temps, mais très vite nous n'avons plus eu le droit de nous faire de signes, et peu après plus personne n'osait. C'était terrible, on ne pouvait pas prendre le risque. »

Du côté bavarois, la frontière commence à devenir un spectacle, le symbole visuel d'un lieu inaccessible où les habitants devinrent isolés. Comme ceci est le cas avec tout symbole, une variété de significations lui est attribuée. À l'Ouest, les habitants disent avoir été témoins de la transformation du paysage frontalier, avoir observé les gardes de l'Est, et évoquent leur adaptation émotionnelle à ce qui était en train de se passer.

\section{Les années 60 et après : " une deuxième nature »}

Alors que la Guerre Froide entre dans une phase d'intensification, le régime frontalier devient alors encore plus strict. En 1970, le gouvernement de la RDA installe des mines terrestres et des mécanismes piégés transformant la démarcation elle-même en piège mortel. Certains des villages que nous avons étudiés sont maintenant masqués par un mur de trois mètres de haut qui sera par la suite doublé à certains endroits. La réglementation concernant la traversée de la frontière est quelque peu allégée vers la fin des années 70 jusqu'au milieu des années 80, mais les déplacements demeurent essentiellement à sens unique alors que la majorité des Allemands de l'Est ne sont pas autorisés à voyager vers l'Ouest. Seuls les retraités ayant à régler d'importantes affaires familiales peuvent voyager en RFA. La zone frontalière immédiate, la « Sperrzone » de 5 $\mathrm{km}$ demeure quant à elle, inaccessible. Les Allemands de l'Ouest peuvent, eux, aller visiter à la journée des destinations spécifiées en RDA et au-delà des zones d'exclusion.

Les récits sur la frontière deviennent bien distincts des deux côtés et développent la notion de «l'autre » : les habitants de l'Ouest adoptent un discours différent de celui des habitants de l'Est, en particulier chez les anciens Allemands de l'Est dont les points de vue varient selon les générations. Les récits «thuringiens » décrivant cette période ne contiennent plus de mémoire visuelle de l'autre côté et qui plus est, deux sphères cognitives d'appartenance semblent devenir réelles pour tous les frontaliers.

Né en 1953, Ralph a grandi dans la zone d'accès limité le long de la démarcation. Selon lui, le mur en face de sa maison de famille et les contrôles quotidiens devinrent une «seconde nature.»

«Hé bien, c'était devenu notre seconde nature. C'était comme cela ici. Pourquoi auriez-vous voulu aller jusqu'à la frontière et molester les gens là-bas [C'est-à-dire les gardes]. Ils faisaient simplement leur travail, leur devoir. Ils n'étaient pas 
responsables. (...) J'ai grandi ici, je connaissais toutes les barrières, le mur, je les voyais tous les jours. Je soudais des équipements à la maison, gaz et oxygène, j'aurais pu couper des morceaux de la barrière et disparaître. Mais pourquoi auraisje voulu prendre un tel risque ? J'avais tout ce dont j'avais besoin ici, ma maison, ma famille et mon travail. Pourquoi donc m'échapper, qu'ils s'en aperçoivent et me tirent dessus dans le dos car c'était leur devoir, car les gardes frontaliers avaient pour ordre de tirer. Et tout ceci pour mourir. Non, non, une telle idée, si j'avais voulu partir, j'y serais tout à fait arrivé. »

31 On se rend compte ici que la frontière est devenue un des aspects de la vie ordinaire de chacun avec lequel on vit au même titre qu'avec n'importe quel autre élément du paysage du village.

Il y a cependant une certaine ambiguïté dans le récit de Ralph : malgré son côté ordinaire, il s'agit tout de même d'une barrière que l'on pouvait violer et traverser si on le voulait, et qui symbolisait une sorte d'Etat absent mais puissant. Il ne précise pas l'origine de cette vision négative, mais il ressent malgré tout le besoin d'y refaire référence : il n'y avait pas de raison valable pour qu'il parte de l'autre côté. Son sentiment d'appartenance est de plus et de toute évidence lié à son côté de la frontière. Il avait là tout ce dont il avait besoin : maison, famille, travail. De façon très intéressante, Ralph ne précise même pas la destination (l'Ouest étant sous-entendu), mais dans tous les cas, cela aurait été un acte stupide et auto-destructeur. Ralph sait aussi pertinemment que cette description de foyer douillet et satisfaisant est contestée par les habitants de l'Ouest, peut-être par d'autres de l'Est, les livres d'Histoire ou les expositions médiatiques. Malgré ceci, il revendique un espace positif.

Les Thuringiens plus âgés décrivent cette période comme ayant transformé leur conception de l'espace : la fermeture de la frontière avait produit le sentiment « d'être enfermé » et fut qualifiée de «terrible ", " étrange », et " amer ». Malgré cela, il y avait aussi l'impression de s'accoutumer progressivement à une situation immuable et de se sentir irrémédiablement attaché à son village.

Du côté bavarois, les diverses visions de la situation sont exprimées dans des discours différents montrant par la même à quel point (Rodman, 1992) les sens donnés à un même paysage géographique peuvent être polysémiques. Du côté bavarois encore une fois, le côté physique de la démarcation se présentait différemment. Les habitants pouvaient s'en rapprocher et même la toucher, pouvaient regarder de l'autre côté et prendre des photos, pouvait la longer en se promenant ou encore converser avec la police frontalière bavaroise en service. La rivière elle-même, sensée être un no man's land entre la RDA et la RFA, demeure un terrain de jeu pour les enfants de l'Ouest. Gelée durant l'hiver, la Saale continue d'être utilisée pour le patin à glace comme elle l'était déjà avant la guerre. Pour les enfants de l'Ouest, l'interdiction « ne jamais toucher le bord de la rivière du côté Est à cause du danger représenté par les gardes frontaliers de la RDA et des mines terrestres, ne devient qu'un autre avertissement parmi ceux déjà existants en rapport avec l'état de la sécurité sur la glace. Pour ceux qui vivaient à l'entour, le paysage frontalier devint bientôt une simple question d'ordinaire et de vie quotidienne. Pour ceux vivant à l'extérieur, en revanche, elle ne cessa jamais d'être un sujet de curiosité stimulée par le frisson de l'inconnu, de la clandestinité et du danger. À l'Ouest, ce sens attribué à la frontière fut maintenu tout au long de son existence à travers ni plus ni moins l'organisation de visites devenues presque rituelles : les locaux conduisaient leurs parents à certains endroits renommés pour leur point de vue, les entreprises avoisinantes amenaient leurs partenaires en affaires en excursion à la 
frontière, les écoles, leurs enfants, et les politiciens, leurs visiteurs étrangers. Les clôtures fortifiées, les tours de garde et les gardes frontaliers en patrouille de la RDA devinrent alors un spectacle de la Guerre Froide, une métaphore de tout ce que l'Ouest n'était pas, et ceci institua un sentiment de supériorité à l'égard de la culture et de l'identité de l'Ouest chez ceux qui pratiquaient cette forme de tourisme en quête de spectacle. De fait, et comme cela devient de plus en plus apparent dans nos entretiens, cette expérience au sens large provoqua des sentiments complexes de pitié, de peur et d'aversion.

De manière évidente pour beaucoup, l'expérience visuelle qu'ils en eurent était la preuve de ce qu'ils savaient déjà des " communistes", des gens radicaux à " $150 \%$ ", qui nourrissaient de l'agressivité contre l'Ouest.

Les récits sur la frontière contiennent de nombreuses interprétations chargées d'émotion, et en particulier lorsque leurs narrateurs se rappellent une traversée de la frontière pour rendre visite à quelqu'un vivant de l'autre côté. L'expérience même du passage des barrières devient celle d'une angoisse incarnée. Karin et Beate sont deux sœurs qui visitèrent la RDA alors qu'elles étaient enfants, et se souviennent ici de cette expérience captivante :

Karin : c'était assez. Oh c'était horrible. A la frontière et oh, c'était horrible

Question : que s'est-il passé à la frontière ?

Karin : tous les contrôles. Ils mettaient des miroirs sous les voitures, et une fois, j'avais tellement besoin d'aller aux toilettes, oh, c'était horrible (elle rit)

Beate : et mère disait toujours : « Soyez sages, ils entendent tout. » (elles rient) vous n'osiez tout simplement rien dire, vous voyez.

Karin : vous vous sentiez totalement observée, un peu comme si quelqu'un vous mettait à nu.

Beate : tout d'un coup, vous vous mettiez à penser que vous aviez commis un crime alors que vous aviez la plus pure des consciences. Pour un enfant...imaginez l'âge que je devais avoir. Vous aviez tellement peur. Il n'est pas étonnant qu'ils (les Allemands de l'Est) aient été si intimidés.

À l'évocation du souvenir d'elles-mêmes dans la voiture de leurs parents, les deux sœurs ressentent à nouveau ce malaise, malgré les rires qu'elles laissent échapper aujourd'hui. Il est intéressant de constater avec quelle force les enfants intériorisèrent ces projections négatives faites sur la RDA, alors fortement répandues et entretenues par le langage courant de la Guerre Froide. Notre base de renseignements regorge de ce genre de référence, et ce, souvent lorsque nos interlocuteurs exagéraient et décrivaient méticuleusement leurs expériences de traversée de la frontière. Ces dernières étaient toujours évaluées en termes d'émotions : avec colère, intimidation, mépris ou sarcasme. Dans ces récits, leurs propres expériences de traversée de la démarcation, ayant été qui plus est légèrement humiliantes, devenaient une représentation du statu quo social et politique de la population est-allemande dans sa totalité.

\section{1989/1990 : la frontière disparaît}

Le 9 septembre 1989, la révolution pacifique de la RDA apporte avec elle la chute du Mur de Berlin. Durant les jours et les semaines suivants, des milliers de citoyens de la 
RDA traversèrent la frontière inter-allemande vers l'Ouest. Hof, la capitale régionale de la Haute Franconie, reçut quotidiennement des milliers de visiteurs. Au moment de la réunification allemande en octobre 1990, beaucoup d'installations ont été démontées et dégagées, et bien souvent avec l'aide de citoyens est-allemands eux-mêmes. Le mur qui séparait le petit village de Mödlareuth est abattu au début du mois de décembre 1989, et une passerelle en bois est officiellement érigée le 31 décembre par les commandants de Hirschberg (Thuringe) et Berg (Bavière) et, avec l'accompagnement de fanfares appartenant aux deux côtés, les habitants mettent un pied de l'autre côté pour la première fois en 40 ans (Stock and Stock 1992).

39 Comme on peut s'y attendre, les récits décrivant cette période sont les plus chargés émotionnellement. Les interlocuteurs sont émus, allant parfois même jusqu'à pleurer à l'évocation de la première traversée de la frontière. Et c'est tout particulièrement le cas des anciens Est-allemands qui se souviennent exactement de ce qu'ils faisaient et de l'endroit où ils se trouvaient lorsqu'ils entendirent la nouvelle de l'ouverture de la frontière et qui le racontent à l'intervieweur (voir aussi Meinhof et Galasinski 2000). Contrastant radicalement avec les récits de Guerre Froide, les habitants des deux côtés de la démarcation se font mutuellement référence sans tonalité idéologique, ils ne sont désormais que des « gens ». De manière significative, et qui plus est, des deux côtés, les habitants décrivent l'espace leur étant auparavant interdit comme un lieu d'activité ; tout le monde traverse et les plus anciens parlent de l'utilisation de carte mentales pour trouver leur chemin de l'autre côté. Les autres n'ayant pas de mémoire du lieu avant la guerre ne reconnaissaient rien et ne savaient pas où ils allaient. Pour beaucoup et quelque soit leur côté d'origine, c'était comme voyager dans l'inconnu.

Linda, née en 1951, ne savait pas comment se rendre chez son cousin a l'Ouest.

«Nous ne savions pas du tout conduire. Il n'y avait pas de carte de l'Ouest. Puis, je me suis souvenue avoir une vieille carte d'école dans le grenier, datant de mes jeunes années à l'école, et alors nous avons conduit sur l'autoroute. Nous n'avions aucune idée du temps que cela prendrait pour aller là-bas. »

41 Contrairement à l'enthousiasme initial ressenti des deux côtés, la liberté de voyager, d'émigrer ou de se rencontrer ne conduisit pas à la réunion familiale harmonieuse ni à l'extinction du processus de marginalisation de «l'autre.» On se rendit alors vite compte que l'on ne trouve pas tout simplement des frères et des sœurs, mais qu'au contraire il fallait les "faire. " Il est intéressant de constater que le symbolique redessin des frontières entre Est et Ouest qui intervint peu de temps après la réunification est aussi décrit en terme de pratiques dans l'espace. Nos interlocuteurs commencèrent alors à construire le territoire de nouveau accessible comme " hostile, " « amical, » « triste, » « agréable » etc. par rapport aux relations économiques et sociales qu'ils commençaient à former et établir.

42 Helga, par exemple, née dans un village frontalier de la Thuringe en 1959, avait des parents habitant de l'autre côté qu'elle vint à connaître après 1989 :

« Hé bien aujourd'hui, parmi mes parents... bon, autrefois vous aviez très envie de leur rendre visite, mais vous ne pouviez pas. Aujourd'hui, je dois dire que c'est assez rare que l'on se voie. Bon, ils viennent pour les anniversaires, mais on ne peut pas dire que cela arrive souvent. Maintenant que l'on peut se voir, cela n'arrive jamais. Peut-être qu'il est devenu normal que l'on reçoive des visites ici aussi désormais. Mais comme je l'ai déjà dit auparavant, ma fille dit toujours que c'est entièrement différent à Munich. Les contacts y sont plus faciles et c'est normal pour eux de vous voir là-bas. Ils n'ont peut-être pas vécu la frontière comme nous, vous voyez. Je ne sais pas vraiment, mais elle dit que là-bas, elle n'a jamais senti, ou rien ne l'a jamais 
poussée à se sentir traitée comme une Ossi (terme péjoratif utilisé pour qualifier les Allemands de l'Est) ». entièrement de son vécu social et de celui de sa famille. Etre traité d'«Ossis " fait référence à un sentiment de supériorité culturelle à laquelle elle fut exposée, et qui plus est par sa famille vivant de l'autre côté de l'ancienne frontière. Dans la lointaine ville de Munich, par contre, sa fille était traitée comme un être humain «normal » et non comme quelqu'un de différent. Ceci détermine donc le concept de lieu et le sentiment d'appartenance d'Helga et qui semble nous informer de son identification à son propre lieu de naissance auquel elle fait référence dans l'entretien. Ce sentiment d'être un stéréotype, comme un être inférieur et d'avoir à se battre contre des visions plus négatives encore sur son lieu de résidence était présent dans les récits de beaucoup d'Allemands de l'Est. L'extrait de Ralph ci-dessus montre un problème identique.

De la même façon, les récits des Allemands de l'Ouest dépeignent un très haut degré d'attachement au lieu de vie. Les représentations du côté Est de la bordure frontalière sont dépeintes de la même façon à travers des récits traitant de relations sociales ou de progrès visibles de l'infrastructure locale. Ces récits vont de celui relatant une nouvelle amitié à celui décrivant un endroit n'ayant que des magasins bon marché à offrir.

\section{Conclusion}

Nous avons montré dans cet article les différentes façons qu'avait la frontière (et quand elles coïncidaient, la rivière de la Saale) d'être présente dans les récits de la vie des individus comme un espace de vie quotidienne tangible, mais aussi comme un symbole fortement visuel de la division et de l'unification de l'Allemagne. Nous avons démontré que les récits qui nous ont été délivrés établissent un lien très étroit entre l'approche personnelle du lieu de vie et le pouvoir à plus grande échelle géographique. Entretenus par des représentations photographiques des différentes phases allant de la période d'avant-guerre à la période post-frontière, tous les récits ont été enregistrés en un entretien/ conversation continu de 70 à 80 minutes. De plus, il est intéressant de relever que les témoignages ne furent pas contraints dans le temps ou dans l'espace des photographies qui elles-mêmes n'avaient pour seul rôle que de fournir des points d'orientation sur lesquels les intervieweurs et les interviewés pouvaient revenir quand ils le voulaient.

Plus de treize ans après la chute du mur de Berlin et douze ans après la (ré)-unification de l'Allemagne, l'importance de cette zone comme (ancienne) ligne de démarcation demeurait indéniable, même pour nos interlocuteurs les plus jeunes ne l'ayant que très peu vécue en tant que frontière effective. En revanche, d'autres générations avaient une façon différente de conceptualiser son importance dans leurs récits du quotidien. Dans chaque entretien, nos interlocuteurs construisirent différents lieux tant géographiques que temporels pour ce qui était leur quotidien, et leur style et leur aisance étaient affectés par le contenu des émotions parfois conflictuelles qui étaient provoquées. Contrairement aux familles réunies de Cetin, la frontière demeure encore aujourd'hui dessinée et présente dans l'esprit des communautés allemandes. Ceci affecte de manière variée la façon de penser et d'évaluer leurs identités chez ceux vivant dans la région de notre étude. 


\section{BIBLIOGRAPHIE}

ARMBRUSTER H. and MEINHOF U.H. (2002). - « Working Identities: Key Narratives in a Former Border Region », in Meinhof U.H. (ed.) Living (with) Borders. Identity Discourses on East-West Borders in Europe. Aldeshot: Ashgate, 15-32.

ARMBRUSTER H. and MEINHOF U.H. (2003) (forthcoming). - « Memories of Home? Narratives of Readjustment on the German/Polish and former German/German Border », in Thornborrow J. and Coates J. (eds.) The Sociolinguistics of Narrative. Amsterdam: John Benjamins.

ARMBRUSTER H., ROLLO C., MEINHOF U.H. (2003) (in press). - « Imagining Europe. Everyday Narratives in European Border Communities ». Journal of Ethnic and Migration Studies, Special Issue, Autumn 2003.

BMIB (Bundesministerium für innderdeutsche Beziehungen) (1989). - Die innerdeutsche Grenze. Bonn.

DonNAN H. and WILSON T. M. (1999). - Borders. Frontiers of Identity, Nation and State. Oxford: Berg. MAIER J. (1990). - « Einführung: Bedeutung und Auswirkungen von Grenzen in Oberfranken zur DDR und CSFR, oder: Wie verändern offene Grenzen einen Raum? », in Maier J. (ed.) Staatsgrenzen und ihr Einfluss auf Raumstrukturen und Verhaltensmuster, Universität Bayreuth, Institut für Geowissenschaften, 1-22.

MASSEY D. (1995). - «The Conceptualization of Place », in Massey D. and Jess P. (eds.) A Place in the World? Places, Cultures and Globalization. Milton Keynes: The Open University, 45-85.

MEINHOF U.H. (ed.) (2002). - Living (with) Borders. Identity Discourses on East-West Borders in Europe, Aldershot: Ashgate.

MEINHOF U.H. and GALASINSKI D. (2000). - « Photography, Memory, and the Construction of Identities on the former East-West German Border », Discourse Studies, 2 (3): 323-353.

MEINHOF U.H. and GALASINSKI D. (2002). - « Reconfiguring East-West identities: cross-generational discourse in German and Polish border communities ", Journal of Ethnic and Migration Studies, 28(1): 63-82.

RODMAN M.C. (1992). - « Empowering Place: Multilocality and Multivocality », American Anthropologist, 94 (3), 640-656.

ROGOFF I. (2000). - Terra Infirma. Geography's Visual Culture. London: Routledge.

sтоск H.F. and sтоск P. (1992). - Auf dem Weg zur Einheit. Vogtland um Hof und Plauen im Brennpunkt. Berlin.

WEBER J. (1990). - « Das Grenzland zwischen Kronach, Plauen, Karlsbad (Karlovy Vary) und Bayreuth - Strukturen und Entwicklungen ", in J. Maier (ed.) Staatsgrenzen und ihr Einfluss auf Raumstrukturen und Verhaltensmuster. Universität Bayreuth: Institut für Geowissenschaften, 23-44.

\section{RÉSUMÉS}

Cet article est basé sur un projet de recherche concernant les identités frontalières le long de l'ancienne frontière intérieure allemande, dans la région de Haute Franconie/ Thuringe et a pour objet la collecte de témoignages. Il éclaire trois périodes de fonctionnement de la frontière qui 
caractérisent en même temps trois phases d'une mentalité frontalière en évolution : la fin des années cinquante au cours desquelles les habitants de la frontière s'habituent lentement à la rupture de leur espace ; les années soixante au cours desquelles la guerre froide définit le statuquo et par là même la délimitation mentale des deux côtés; et enfin la période 1989-1990 qui certes efface la frontière, mais ne produit pas de nouvelle solidarité. L'article discute de la façon dont ces périodes sont évoquées et représentées dans les témoignages. On étudie en particulier la signification de ce qui relie presque charnellement l'individu à une région. En effet, la frontière a d'abord été vécue comme une barrière physique insurmontable coupant une région autrefois unifiée et a changé la vie des gens d'une manière souvent dramatique. Mais la région frontalière a aussi toujours été à la fois un espace tangible aux contours bien délimités et une carte mentale, produit des représentations et des expériences individuelles. En observant la signification de la région frontalière dans les récits des gens, on étudie la relation entre l'espace physique et culturel et on montre dans quelle mesure le vécu de la transformation historique est relaté comme faisant partie de la connaissance de la région.

This chapter is based on our study of Border identities on the former German-German border in the region of northern Bavaria/southern Thuringia. It discusses narratives about three historical phases of border definition: The late 1950s which saw a gradual coming to terms with division on both sides; the 1960s onwards where the Cold War confirmed the status quo for people on either side, and the period after 1989/90, which led to a dissolution of the sociopolitical division with the fall of the wall, but without necessarily creating a new sense of togetherness. We explore the significance of landscape and embodied knowledge in our data. Evidently, the border we are concerned with was a virtually unsurmountable physical construct which cut through a once unified cultural region and changed people's lives in often dramatic ways. The border landscape was always both: an item of tangible geography and a cultural and cognitive map, both shaping and constraining people's experiences and personal histories. By looking at the significance of the border landscape in people's narratives we investigate the relationship between the physical and the cultural, and elucidate in how far the experience of historical transformation is narrated as embodied spatial knowledge.

Dieser Artikel beruht auf einem Forschungsprojekt über Grenzidentitäten an der ehemaligen deutsch-deutschen Grenze im Raum Oberfranken / Thüringen und hat Interviewnarrationen zum Gegenstand. Er beleuchtet drei Phasen der Grenzziehung, die zugleich drei Phasen einer sich verändernden Grenzmentalität kennzeichnen: Die späten 50iger Jahre, in der GrenzbewohnerInnen sich langsam an die Tatsache eines durchschnittenen Lebensraumes gewöhnen; die 60iger Jahre, in der der kalte Krieg den statu-quo und damit die mentale Abgrenzung beider Seiten definiert, und schliesslich die Periode nach 1989-90, die zwar die Grenze aufhebt aber keine neue Solidarität erzeugt. Der Artikel diskutiert wie diese Phasen in den Interviewdaten erinnert und repräsentiert werden. Aspekte von Landschaft und Verkörperlichung spielen dabei eine besondere Rolle, da die Grenze immer beides war: eine undurchdringbare physische Mauer und eine soziokulturelle und kognitive Tangente. Der Artikel zeigt, wie das Ineinanderverwobensein der physischen und soziokulturellen Dimension von Landschaft Selbstverständnis und historische Erfahrung der Grenzbevölkerung geformt hat. 
INDEX

Mots-clés : frontière entre la RDA et la RFA, Haute-Franconie, histoire orale, identités spatiales, populations frontalières, Thuringe

Schlüsselwörter : Deutsch-Deutsche Grenze, Grenzbevölkerungen, Oberfranken, oral history, räumliche Identiäten, Saale, Thüringen

Keywords : border populations, German-German border, oral history, Saale, spatial identities, Thuringia, Upper Franconia 